\title{
Feasibility and speed of insertion of seven supraglottic airway devices under simulated airway conditions
}

\author{
Oliver Robak, MD*; Marco Leonardelli, MD*; Konstantin Zedtwitz-Liebenstein, $\mathrm{MD}^{\dagger}$; \\ Kurt Rützler, $\mathrm{MD}^{\ddagger}$; Ernst Schuster, $\mathrm{PhD}^{\S}$; Sonia Vaida, MD"; Ramez Salem, MD ${ }^{\uparrow}$; Michael Frass, MD
}

\section{ABSTRACT}

Objectives: Endotracheal intubation (ETI) is considered the gold standard for protecting the airway. Alternative devices for airway protection have been developed that can be used by untrained personnel, by those with less experience, and for when ETI is not possible. The main goals of our study were to evaluate the success rate and speed of insertion of different supraglottic airway devices and to determine whether the devices could be properly inserted under simulated critical conditions.

Methods: Fifty medical students used an airway simulation trainer (Laerdal SimMan 3G) to assess the success rate and time used to insert seven different supraglottic airway devices under simulated physiologic and pathologic conditions in two different runs.

Results: Although all airway devices could be inserted without problems, only the Combitube and the EasyTube could be successfully inserted in simulations of trismus, limited mobility of the cervical spine, or a combination of pathologic conditions such as trismus plus limited mobility of the spine and trismus plus tongue edema. The insertion time was significantly longer with LMA Unique, Fastrach, and I-Gel devices in both the first and second runs.

Conclusion: The Combitube and the EasyTube were most easily inserted under simulated conditions such as trismus, limited mobility of the cervical spine, and combined pathologic conditions. Although all devices are useful for establishing an airway by nontrained medical students in standard simulations, we suggest that the Combitube and the EasyTube may offer advantages in difficult airway situations.

\section{RÉSUMÉ}

Objectifs: Le tube endotrachéal est considéré comme le moyen par excellence de protection des voies aériennes. Cependant, il existe d'autres dispositifs de protection des voies aériennes qui peuvent être utilisés par du personnel non formé ou des personnes ayant peu d'expérience ou encore dans des cas où l'intubation endotrachéale n'est pas possible. L'étude avait pour buts principaux d'évaluer le taux de réussite de la mise en place et le temps de pose de différents dispositifs supraglottiques et de déterminer si ces dispositifs pouvaient être installés correctement dans des situations d'urgence simulées.

Méthodes: Cinquante étudiants en médecine ont utilisé un simulateur de voies aériennes (Laerdal SimMan 3G) pour évaluer le taux de réussite de la mise en place et le temps de pose de sept dispositifs supraglottiques différents, dans des états physiologiques et pathologiques simulés, en deux séances.

Résultats: II a été possible de poser tous les dispositifs sans problème, mais seuls les tubes Combitube et EasyTube ont pu être mis en place dans des états simulés de trismus, de mobilité réduite de la colonne cervicale, ou d'association d'états pathologiques tels que le trismus et une mobilité réduite de la colonne cervicale ou le trismus et un oedème de la langue. Le temps de mise en place d'autres dispositifs: le LMA Unique, le Fastrach, et I'I-Gel, était passablement plus long, et ce, au cours de la première et de la deuxième séance. Conclusion: Les tubes Combitube et EasyTube ont été les dispositifs les plus faciles à mettre en place dans les conditions simulées de trismus, de mobilité réduite de la colonne cervicale, ou d'association d'états pathologiques. Bien que tous les dispositifs soient utiles dans le rétablissement de la perméabilité des voies aériennes par des étudiants en médecine, non formés, dans des conditions normales de simulation, nous estimons que les tubes Combitube et EasyTube peuvent se montrer avantageux dans des situations difficiles de ventilation.

Keywords: emergency medicine, manikin, supraglottic airway

From the *Department of Medicine I, Medical University Vienna, Vienna, Austria; †Head Office of Vienna Hospital Association, Vienna, Austria; $\ddagger$ Department of Anesthesiology and General Intensive Care, Medical University Vienna, Vienna, Austria; §Center for Medical Statistics, Informatics and Intelligent Systems, Medical University Vienna, Vienna, Austria; || Department of Anesthesiology, Penn State Milton S. Hershey Medical Center, Hershey, PA; ๆDepartment of Anesthesiology, Advocate Illinois Masonic Medical Center, Chicago, IL.

Correspondence to: Dr. Oliver Robak, Department of Medicine I, Intensive Care Unit, Medical University Vienna, Waehringer Guertel 18-20, A 1090 Vienna, Austria; oliver.robak@meduniwien.ac.at.

This article has been peer reviewed. 
Securing the airway in an emergency is a pivotal skill for all health care providers. Endotracheal intubation (ETI) is considered the gold standard for securing an airway. This technique requires specific motor skills and frequent practice to be performed quickly and safely. ${ }^{1,2}$ The European Resuscitation Council (ERC) guidelines for cardiopulmonary resuscitation (CPR) (2010) recommend that ETI be performed by experienced and trained personnel only. ${ }^{3}$ Many health care providers do not have the requisite knowledge and practice to safely and quickly perform ETI. For these providers, supraglottic devices provide a valid alterative. These devices do not require laryngoscopy. Although they should not be used to overcome glottis or subglottic airway obstruction, they are useful in a variety of emergencies when ventilation is required.

Critically ill patients are at risk for adverse airwayrelated problems. ${ }^{4}$ Airway-related mortality rates range from 5 to $8 \%{ }^{4}$ Clinicians are responsible for up to $50 \%$ of these adverse events, with less experienced physicians or inadequately trained personnel often involved. ${ }^{5}$ Given that, as a group, medical students are not considered experienced in ETI or airway management, ${ }^{6,7}$ we chose a cohort of students to evaluate the efficacy of various supraglottic airway devices in select pathologic situations. We used a manikin to standardize the clinical scenarios. ${ }^{8}$

The primary goal of our study was to evaluate the feasibility of insertion of different supraglottic airway devices under simulated physiologic and pathologic conditions. Our secondary goal was to measure the time to successful insertion. Additionally, we compared the results of the first and second runs.

\section{METHODS}

Fifty medical students $(\mathrm{M}: \mathrm{F}=1: 1)$ in their third to fifth year participated in this study. Students did not have any previous experience in airway management. The Ethical Committee of the Medical University Vienna, Austria, approved the study. The participants signed informed consent forms prior to the study, agreeing to participate in two separate runs with the respective supraglottic devices. The study was conducted between March and September 2010.

We used an airway simulation manikin (Laerdal SimMan 3G, Laerdal, Stavanger, Norway) to assess the feasibility of insertion and the insertion time of seven airway devices (LMA Unique, Fastrach single use, and LMA Supreme, all LMA Company North America, San
Diego, CA; laryngeal tube single use King-LTS-D, VBM, Sulz, Germany; I-Gel, Intersurgical Ltd., Wokingham, UK; Combitube, Covidien, Mansfield, MA; and EasyTube, Teleflexmedical Ruesch, Research Triangle Park, NC). Two experienced medical professionals (O.R., M.L.) assessed the correct placement using a laryngoscope and the insertion time using a stopwatch. We measured the time from grasping the device to final placement. If necessary, cuffs were inflated and the manikin was ventilated to confirm successful placement of the airway device. Insertion attempts lasting for more than 30 seconds were classified as unsuccessful.

First, intubation under simulated physiologic airway conditions was performed. Then simulated pathologic airway conditions such as trismus, limited mobility of the cervical spine, tongue edema, and combinations of two conditions (trismus and limited mobility of the spine, trismus and tongue edema) were simulated to aggravate insertion of the airway devices. The order of the devices and the conditions were randomized by a computergenerated code. Each student performed all six scenarios (one physiologicaand five pathologic situations) with each of the seven devices. Therefore, each student performed 42 insertions during each of the two runs. Randomization was prepared in a sealed envelope for each participant. The devices were explained for only 20 seconds each prior to use as we considered the use of the airway devices self-explanatory. The time needed for insertion was assessed (first run) and reassessed in the same setting 3 months later (second run). First and second runs were analyzed separately and compared to each other. The computer-generated code was held until the end of the study, and the results were forwarded in a blinded manner to the statistician. The code was broken only after statistical evaluation.

The sample size calculation was based on a type I error alpha of 0.008 corrected for multiple testing, a power of 0.9 , a mean difference of 1.0 , and a standard deviation (SD) within the treatment groups of sigma $=$ 1.5 , resulting in a sample size of 38 . With an estimated dropout rate of $20 \%$ during the second run, we enrolled 50 students. For analysis of differences between the first and the second run and of differences between the standard physiologic and the pathologic conditions, we used the paired $t$-test with correction for repeated measurements. For analysis of differences between the various types of supraglottic airway devices, we used the general linear model (GLM) procedure for repeated measurements. The results are 
expressed as mean $\pm \mathrm{SD}$. A $p$ value of less than 0.05 was considered significant.

\section{RESULTS}

Five students did not show up during the second part of the study and therefore were excluded from the study. Forty-five students completed the first and second parts of the study; 25 were female (55.6\%). Ages ranged from 19 to 45 years (mean age $26.57 \pm 6.42$ years).

\section{Success rate and speed of insertion of first and second runs using seven devices under simulated physiologic airway conditions}

Under simulated physiologic conditions, all devices could be inserted successfully in both runs on the first attempt. When the first and second runs were compared for each device, significantly shorter insertion times during the second run could be found with the LMA Unique and the Fastrach under physiologic conditions (Table 1).

\section{Success rate and speed of insertion of first and second run using seven devices under simulated pathologic airway conditions}

All devices could be inserted in the simulated situation of tongue edema. However, only the Combitube and the Easy Tube could be successfully inserted in trismus, limited mobility of the cervical spine, and combined pathologic conditions such as trismus plus limited mobility of the spine and trismus plus tongue edema. In the simulated conditions of tongue edema, the LMA Unique, Fastrach, and Supreme were inserted significantly faster during the second run when compared to the first run (Table 2).

With regard to device and condition (physiologic or pathologic), there was a significant time differencebut of questionable clinical significance-between the first and second runs (7.2 v. 6.0 seconds, $p<0.05$ ).

\begin{tabular}{|c|c|c|c|}
\hline Device & First run & Second run & Paired t-test, $p$ \\
\hline LMA Unique & $6.11 \pm 2.6$ & $5.11 \pm 1.65$ & 0.0281 \\
\hline Fastrach & $5.91 \pm 5.04$ & $4.17 \pm 1.58$ & 0.0161 \\
\hline Supreme & $4.89 \pm 1.96$ & $4.49 \pm 1.70$ & 0.2853 \\
\hline King-LTS-D & $6.58 \pm 2.83$ & $6.52 \pm 2.71$ & 0.9096 \\
\hline I-Gel & $6.93 \pm 2.13$ & $6.33 \pm 2.57$ & 0.1494 \\
\hline Combitube & $7.49 \pm 6.36$ & $5.87 \pm 3.27$ & 0.1217 \\
\hline EasyTube & $4.64 \pm 2.02$ & $5.07 \pm 2.03$ & 0.3159 \\
\hline
\end{tabular}

\begin{tabular}{|c|c|c|c|}
\hline Device & First run & Second run & Paired $t$-test, $p$ \\
\hline LMA Unique* & $9.44 \pm 8.71$ & $6.80 \pm 5.99$ & 0.0116 \\
\hline Fastrach* & $15.31 \pm 13.13$ & $10.35 \pm 10.91$ & 0.0030 \\
\hline Supreme* & $5.20 \pm 2.46$ & $4.38 \pm 1.42$ & 0.0431 \\
\hline King-LTS-D* & $7.44 \pm 4.94$ & $8.89 \pm 6.42$ & 0.1241 \\
\hline $\mid-\mathrm{Gel}^{*}$ & $11.51 \pm 9.91$ & $9.22 \pm 7.91$ & 0.1002 \\
\hline Combitube $^{\dagger}$ & $6.24 \pm 4.71$ & $4.98 \pm 1.91$ & 0.0538 \\
\hline EasyTube $^{\dagger}$ & $4.82 \pm 2.14$ & $4.71 \pm 1.80$ & 0.4082 \\
\hline
\end{tabular}

\section{Combitube and EasyTube under simulated pathologic conditions}

Given that only the Combitube and the EasyTube could be inserted in trismus, limited spine mobility, and combined pathologic conditions, those two devices were compared in a subgroup analysis. With regard to speed of insertion, there was a significant difference between the Combitube and the EasyTube (7.3 v. 5.9 seconds, $p<0.05)$.

\section{Comparison between simulated physiologic and pathologic airway conditions}

When physiologic and pathologic conditions were compared during the first run, a significantly longer insertion time was needed with the LMA Unique, Fastrach, and I-Gel (Table 3). Comparison of physiologic and pathologic conditions during the second run showed similar results with significant prolonged time to insert the LMA Unique, Fastrach, King-LTS-D, and I-Gel in simulated pathologic conditions (Table 4).

Table 3. First run: comparison between physiologic and pathologic conditions (seconds, mean \pm SD)

\begin{tabular}{|lccc|}
\hline Device & Physiologic & Pathologic & Paired t-test, $p$ \\
\hline LMA Unique & $6.11 \pm 2.6$ & $9.44 \pm 8.71^{*}$ & 0.0076 \\
Fastrach & $5.91 \pm 5.04$ & $15.31 \pm 13.13^{*}$ & $<0.0001$ \\
Supreme & $4.89 \pm 1.96$ & $5.20 \pm 2.46^{*}$ & 0.3152 \\
King-LTS-D & $6.58 \pm 2.83$ & $7.44 \pm 4.94^{*}$ & 0.1768 \\
I-Gel & $6.93 \pm 2.13$ & $11.51 \pm 9.91^{*}$ & 0.0023 \\
Combitube & $7.49 \pm 6.36$ & $6.24 \pm 4.71^{\dagger}$ & 0.0573 \\
EasyTube & $4.64 \pm 2.02$ & $4.82 \pm 2.14^{\dagger}$ & 0.5569 \\
\hline
\end{tabular}


Whereas the Combitube and the Easy'Tube could be inserted in all of the five investigated pathologic conditions, the other five airway devices could be evaluated only in tongue edema because the other pathologic conditions prevented insertion. When an overall analysis was performed, insertion took longer under pathologic conditions than in physiologic conditions $(5.71$ v. 7.82 seconds, $p<0.05)$. There was a small training effect with a shorter insertion time during the second run compared to the first run $(7.32$ v. $6.21, p<0.05)$.

\section{Adverse effects}

With regard to adverse effects, the handle of one Fastrach device broke during the trial. No other adverse effects were observed.

\section{DISCUSSION}

The current study shows that all devices could be inserted effectively and rapidly under simulated physiologic conditions. We observed a significant difference between the various supraglottic airway devices with regard to speed of insertion under physiologic and pathologic conditions. This proves that the learning effect is device specific. We also observed that during the second run, most participants were able to insert the device significantly faster compared to the first run, suggesting a training effect; however, the observed mean time difference is clinically not relevant.

A major limitation of the study is the use of a manikin instead of real patients. However, manikins are very similar to human anatomy and allow for simulating the same airway situation for each participant. Also, the manikin's anatomy may favour a specific model of airway device. ${ }^{9}$ To rule out this bias, further

Table 4. Second run: comparison between physiologic and pathologic conditions (seconds, mean \pm SD)

\begin{tabular}{lccc|}
\hline Device & Physiologic & Pathologic & Paired $t$-test, $p$ \\
\hline LMA Unique & $5.11 \pm 1.65$ & $6.80 \pm 5.99^{*}$ & 0.0393 \\
Fastrach & $4.17 \pm 1.58$ & $10.35 \pm 10.91^{*}$ & 0.0012 \\
Supreme & $4.49 \pm 1.70$ & $4.38 \pm 1.42^{*}$ & 0.6135 \\
King-LTS-D & $6.52 \pm 2.71$ & $8.89 \pm 6.42^{*}$ & 0.0112 \\
I-Gel & $6.33 \pm 2.57$ & $9.22 \pm 7.91^{*}$ & 0.0146 \\
Combitube & $5.87 \pm 3.27$ & $4.98 \pm 1.91^{\dagger}$ & 0.2425 \\
EasyTube & $5.07 \pm 2.03$ & $4.71 \pm 1.80^{\dagger}$ & 0.5178 \\
\hline *Only tongue edema investigated. & & \\
'All five pathologic conditions investigated. & & \\
\hline
\end{tabular}

studies using different models of manikins are warranted. Another limitation is that every attempt lasting for more than 30 seconds was considered a failure, although it might have eventually been successful. This is in accordance with the guidelines of the ERC, which now recommend aborting intubation attempts lasting more than 10 seconds ${ }^{10}$ because persistent and prolonged intubation attempts may lead to a fatal outcome. ${ }^{11}$ The ability to offer an alternative to ETI is pivotal.

Wang and colleagues showed that ETI efforts in out-of-hospital cardiac arrests led to prolonged interruptions of CPR. ${ }^{12}$ As the time to ventilation is shorter when using supraglottic airway devices, ${ }^{13,14}$ particularly in difficult cases, ${ }^{15}$ it seems reasonable to perform a prospective trial comparing the placement of supraglottic devices to that of conventional endotracheal tubes, investigating the time needed for interrupting heart massage during $\mathrm{CPR}$, and recognizing that airway management does not alter outcomes in patients in cardiac arrest.

The main finding of the study is that only the Combitube and the EasyTube could be inserted under all simulated pathologic conditions. For example, with trismus, limited mobility of the cervical spine, and combined pathologic conditions (trismus and limited mobility of the spine, trismus and tongue edema), insertion of all the LMA devices, the King-LTS-D, and the I-Gel was unsuccessful without breaking the teeth of the manikin due to the small interincisor distance and the small oral cavity. This may be relevant in choosing a rescue airway device, but care must be taken in translating simulation findings to clinical situations.

Levitan and colleagues recommended conducting a study that would evaluate whether a laryngeal mask airway can be placed by medical personnel with limited or no laryngoscopy skills. ${ }^{16}$ Our study shows that it is possible for inexperienced medical students to secure an airway in a manikin with different supraglottic airway devices. We therefore agree with Wahlen and colleagues, who recommended the use of supraglottic devices in situations where only limited airway training is possible. ${ }^{17}$ Furthermore, a training effect was maintained over a period of 3 months: participants showed even faster insertion times at the second time point $(p<0.05)$.

Pathologic conditions such as trismus and limited mobility of the spine, and combined pathologic conditions could be successfully tackled only with the use of the Combitube or the EasyTube. Although trismus may be rare, a similar condition is found in 
clenched jaw. It has been shown by Bollig and colleagues that the time to successful ventilation was longer for conventional ETI compared to the Combitube and the EasyTube, as used by paramedics, although there was no significant time difference between the Combitube and the EasyTube. ${ }^{14}$ Further advantages of the Combitube and the EasyTube are that only one size is needed for most adults.

Other simulation studies have shown that insertion of the laryngeal tube was easier than insertion of the Combitube..$^{18,19}$ These studies were done without any pathologic condition simulated by the manikin, which limits their generalization. The superiority of supraglottic airway devices to bag-valve ventilation was recently shown by Fischer and colleagues. ${ }^{20}$

\section{CONCLUSION}

Supraglottic airway devices are commonly used as an alternative to endotracheal tubes. We investigated the feasibility and time of insertion of different airway devices by untrained medical students. All devices can be used to establish an airway by untrained medical students under physiologic conditions, but the Combitube and the EasyTube provide advantages compared to the other supraglottic airway devices.

Competing interests: Michael Frass has received royalties from Covidien in the past.

\section{REFERENCES}

1. Deakin CD, King P, Thompson F. Prehospital advanced airway management by ambulance technicians and paramedics: is clinical practice sufficient to maintain skills? Emerg Med f 2009;26:888-91, doi:10.1136/emj.2008.064642.

2. Lecky F, Bryden D, Little R, et al. Emergency intubation for acutely ill and injured patients. Cochrane Database Syst Rev 2008;(2):CD001429.

3. Nolan JP, Soar J, Zideman DA, et al. European Resuscitation Council Guidelines for Resuscitation 2010 Section 1. Executive summary. Resuscitation 2010;81:121976, doi:10.1016/j.resuscitation.2010.08.021.

4. Wilson RM, Runciman WB, Gibberd RW, et al. The Quality in Australian Health Care Study. Med 7 Aust 1995; $163: 458-71$.

5. Wu AW, Folkman S, McPhee SJ, et al. Do house officers learn from their mistakes? FAMA 1991;265:2089-94, doi:10.1001/jama.1991.03460160067031.

6. Perkins GD, Barrett H, Bullock I, et al. The Acute Care Undergraduate TEaching (ACUTE) Initiative: consensus development of core competencies in acute care for undergraduates in the United Kingdom. Intensive Care Med 2005; 31:1627-33, doi:10.1007/s00134-005-2837-4.
7. Smith CM, Perkins GD, Bullock I, et al. Undergraduate training in the care of the acutely ill patient: a literature review. Intensive Care Med 2007;33:901-7, doi:10.1007/ s00134-007-0564-8.

8. MacDowall J. The assessment and treatment of the acutely ill patient - the role of the patient simulator as a teaching tool in the undergraduate programme. Med Teach 2006;28: 326-9, doi:10.1080/01421590600625130.

9. Jackson KM, Cook TM. Evaluation of four airway training manikins as patient simulators for the insertion of eight types of supraglottic airway devices. Anaesthesia 2007;62:388-93, doi:10.1111/j.1365-2044.2007.04983.x.

10. Nolan JP, Deakin CD, Soar J, et al. European Resuscitation Council guidelines for resuscitation 2005. Section 4. Adult advanced life support. Resuscitation 2005;67 Suppl 1:S39-86, doi:10.1016/j.resuscitation.2005.10.009.

11. Caplan RA, Posner KL, Ward RJ, et al. Adverse respiratory events in anesthesia: a closed claims analysis. Anesthesiology 1990;72:828-33, doi:10.1097/00000542-199005000-00010.

12. Wang HE, Simeone SJ, Weaver MD, et al. Interruptions in cardiopulmonary resuscitation from paramedic endotracheal intubation. Ann Emerg Med 2009;54:645-52 e641, doi: 10.1016/j.annemergmed.2009.05.024.

13. Bein B, Carstensen S, Gleim M, et al. A comparison of the proseal laryngeal mask airway, the laryngeal tube $S$ and the oesophageal-tracheal Combitube during routine surgical procedures. Eur $\mathcal{F}$ Anaesthesiol 2005;22:341-6, doi:10.1017/ S026502150500058X.

14. Bollig G, Lovhaug SW, Sagen O, et al. Airway management by paramedics using endotracheal intubation with a laryngoscope versus the oesophageal tracheal Combitube and EasyTube on manikins: a randomised experimental trial. Resuscitation 2006;71:107-11, doi:10.1016/j.resuscitation.2006. $\underline{02.016 .}$

15. Rabitsch W, Schellongowski $\mathrm{P}$, Staudinger $\mathrm{T}$, et al. Comparison of a conventional tracheal airway with the Combitube in an urban emergency medical services system run by physicians. Resuscitation 2003;57:27-32, doi:10.1016/ S0300-9572(02)00435-5.

16. Levitan RM, Ochroch EA, Stuart S, et al. Use of the intubating laryngeal mask airway by medical and nonmedical personnel. Am 7 Emerg Med 2000;18:12-6, doi:10.1016/ S0735-6757(00)90040-8.

17. Wahlen BM, Roewer N, Lange M, et al. Tracheal intubation and alternative airway management devices used by healthcare professionals with different level of pre-existing skills: a manikin study. Anaesthesia 2009;64:549-54, doi:10.1111/ j.1365-2044.2008.05812.x.

18. Huter L, Schwarzkopf K, Rodiger J, et al. Students insert the laryngeal tube quicker and more often successful than the esophageal-tracheal combitube in a manikin. Resuscitation 2009;80:930-4, doi:10.1016/j.resuscitation.2009.04.038.

19. Trabold B, Schmidt C, Schneider B, et al. Application of three airway devices during emergency medical training by health care providers-a manikin study. Am 7 Emerg Med 2008;26:783-8, doi:10.1016/j.ajem.2007.11.006.

20. Fischer H, Hochbrugger E, Fast A, et al. Performance of supraglottic airway devices and 12 month skill retention: a randomized controlled study with manikins. Resuscitation 2011;82:326-31. 